\title{
A new method for mathematical and simulation modelling interactivity: A case study in flexible job shop scheduling
}

\author{
Ojstersek, R..$^{a,}{ }^{,}$, Lalic, D. ${ }^{b}$, Buchmeister, B. ${ }^{a}$ \\ ${ }^{a}$ University of Maribor, Faculty of Mechanical Engineering, Maribor, Slovenia \\ ${ }^{b}$ University of Novi Sad, Faculty of Technical Sciences, Novi Sad, Serbia
}

\begin{abstract}
A B S T R A C T
The present study has investigated mathematical and simulation model interactivity for production system scheduling. A mathematical model of a Flexible Job Shop Scheduling Production optimisation problem (FJSSP) was used to evaluate a new evolutionary computation method of multi-objective heuristic Kalman algorithm (MOHKA). Ten Brandimarte and five Kacem benchmarks were applied for evaluation and comparison of MOHKA optimisation results with the Multi-Objective Particle Swarm Optimization algorithm (MOPSO) and Bare-Bones Multi-Objective Particle Swarm Optimization algorithm (BBMOPSO). Benchmark data sets were divided into three groups, regarding their complexity, from low, middle to high dimensional optimisation problems. The optimisation results of MOHKA show high capability to solve complex multiobjective optimisation problems, especially with real world production systems data. A new robust method is presented of optimisation data interactivity between a mathematical optimisation algorithm and a simulation model. The results show that the presented method can overcome the integrated decision logic of commercial simulation software and transfer the optimisation results into the simulation model. Our interactive method can be used in a variety of production and service companies to ensure an optimised and sustainable cost-time profile.
\end{abstract}

(C) 2019 CPE, University of Maribor. All rights reserved.

\begin{tabular}{l} 
A R T I C L E I N F O \\
\hline Keywords: \\
Flexible job shop scheduling; \\
Mathematical modelling; \\
Simulation modelling; \\
Interactivity; \\
Evolutionary computation; \\
Multi-objective heuristic Kalman \\
algorithm (MOHKA); \\
Multi-objective particle swarm \\
optimization (MOPSO); \\
Bare-bones multi-objective parti- \\
cle swarm optimization algorithm \\
(BBMOPSO) \\
*Corresponding author: \\
robert.ojstersek@um.si \\
(Ojstersek, R.) \\
Article history: \\
Received 15 May 2019 \\
Revised 21 November 2019 \\
Accepted 7 December 2019
\end{tabular}

\section{References}

[1] Schwab, K. (2017). The fourth industrial revolution, World Economic Forum, New York, USA.

[2] Li, Y., Yao, X., Zhou, J. (2016). Multi-objective optimization of cloud manufacturing service composition with cloud-entropy enhanced genetic algorithm, Strojniški Vestnik - Journal of Mechanical Engineering, Vol. 62, No. 10, 577-590, doi: $10.5545 /$ sv-jme.2016.3545.

[3] Chaudhry, I.A., Usman, M. (2017). Integrated process planning and scheduling using genetic algorithms, Tehnički Vjesnik - Technical Gazette, Vol. 24, No. 5, 1401-1409, doi: 10.17559/TV-20151121212910.

[4] Huang, J., Süer, G.A. (2015). A dispatching rule-based genetic algorithm for multi-objective job shop scheduling using fuzzy satisfaction levels, Computers \& Industrial Engineering, Vol. 86, 29-42, doi: 10.1016/i.cie.2014. 12.001.

[5] Mitchell, M. (1998). An introduction to genetic algorithms, MIT press, Cambridge, England.

[6] Hao, X., Gen, M., Lin, L., Suer, G.A. (2017). Effective multiobjective EDA for bi-criteria stochastic job-shop scheduling problem, Journal of Intelligent Manufacturing, Vol. 28, No. 3, 833-845, doi: 10.1007/s10845-0141026-0.

[7] Meolic, R., Brezočnik, Z. (2018). Flexible job shop scheduling using zero-suppressed binary decision diagrams, Advances in Production Engineering \& Management, Vol. 13, No. 4, 373-388, doi: 10.14743/apem2018.4.297.

[8] Wang, H., Wang, W., Sun, H., Cui, Z., Rahnamayan, S., Zeng, S. (2017). A new cuckoo search algorithm with hybrid strategies for flow shop scheduling problems, Soft Computing, Vol. 21, No. 15, 4297-4307, doi: 10.1007/s00500- 
016-2062-9.

[9] Vujica Herzog, N., Buchmeister, B., Beharic, A., Gajsek, B. (2018). Visual and optometric issues with smart glasses in Industry 4.0 working environment, Advances in Production Engineering \& Management, Vol. 13, No. 4, 417 428, doi: $10.14743 /$ apem2018.4.300.

[10] Zhang, H., Liu, S., Moraca, S., Ojstersek, R. (2017). An effective use of hybrid metaheuristics algorithm for job shop scheduling problem, International Journal of Simulation Modelling, Vol. 16, No. 4, 644-657, doi: 10.2507/ IJSIMM16(4)7.400.

[11] Fu, H.C., Liu, P. (2019). A multi-objective optimization model based on non-dominated sorting genetic algorithm, International Journal of Simulation Modelling, Vol. 18, No. 3, 510-520, doi: 10.2507/IJSIMM18(3)C012.

[12] Gotlih, J., Brezocnik, M., Balic, J., Karner, T., Razborsek, B., Gotlih, K. (2017). Determination of accuracy contour and optimization of workpiece positioning for robot milling, Advances in Production Engineering \& Management, Vol. 12, No. 3, 233-244, doi: 10.14743/apem2017.3.254.

[13] Pantić, M., Đorđević, A., Erić, M., Mitrović, S., Babić, M., Džunić, D., Stefanović, M. (2018). Application of artificial neural network in biotribological research of dental glass ceramic, Tribology in Industry, Vol. 40, No. 4, 692-701, doi: $10.24874 /$ ti.2018.40.04.15.

[14] Ojstersek, R., Zhang, H., Liu, S., Buchmeister, B. (2018). Improved heuristic kalman algorithm for solving multiobjective flexible job shop scheduling problem, Procedia Manufacturing, Vol. 17, 895-902, doi: 10.1016/i.promfg. 2018.10.142.

[15] Rupnik, B., Nardin, R., Kramberger, T. (2019). Discrete event simulation of hospital sterilization logistics, Tehnički Vjesnik - Technical Gazette, Vol. 26, No. 5, 1486-1491, doi: 10.17559/TV-20180614102011.

[16] Kacem, I., Hammadi, S., Borne, P. (2002). Pareto-optimality approach for flexible job-shop scheduling problems: Hybridization of evolutionary algorithms and fuzzy logic, Mathematics and Computers in Simulation, Vol. 60, No. 3-5, 245-276, doi: 10.1016/S0378-4754(02)00019-8.

[17] Mastrolilli, M., Gambardella, L.M. (2000). Effective neighbourhood functions for the flexible job shop problem, Journal of Scheduling, Vol. 3, No. 1, 3-20, doi: 10.1002/(SICI)1099-1425(200001/02)3:1<3::AID-JOS32>3.0.CO;2$\underline{\mathrm{Y}}$.

[18] Mostaghim, S., Teich, J. (2003). Strategies for finding good local guides in multi-objective particle swarm optimization (MOPSO), In: Proceedings of the 2003 IEEE Swarm Intelligence Symposium, Indianapolis, USA, 26-33, doi: $10.1109 /$ SIS.2003.1202243.

[19] Zhang, Y., Gong, D.-W., Ding, Z. (2012). A bare-bones multi-objective particle swarm optimization algorithm for environmental/economic dispatch, Information Sciences, Vol. 192, 213-227, doi: 10.1016/j.ins.2011.06.004.

[20] Cajzek, R., Klanšek, U. (2016). Mixed-integer nonlinear programming based optimal time scheduling of construction projects under nonconvex costs, Tehnički Vjesnik - Technical Gazette, Vol. 23, No. 1, 9-18, doi: $10.17559 / \mathrm{TV}-20140108112928$.

[21] Becker, C., Scholl, A. (2009). Balancing assembly lines with variable parallel workplaces: Problem definition and effective solution procedure, European Journal of Operational Research, Vol. 199, No. 2, 359-374, doi: 10.1016/ j.ejor.2008.11.051.

[22] Graham, R.L., Lawler, E.L., Lenstra, J.K., Kan Rinnooy, A.H.G. (1979). Optimization and approximation in deterministic sequencing and scheduling: A survey, Annals of Discrete Mathematics, Vol. 5, 287-326, doi: 10.1016/S0167-5060(08)70356-X.

[23] Lin, L., Gen, M. (2018). Hybrid evolutionary optimisation with learning for production scheduling: State-of-theart survey on algorithms and applications, International Journal of Production Research, Vol. 56, No. 1-2, 193223, doi: $10.1080 / 00207543.2018 .1437288$.

[24] Miettinen, K.M. (2012). Nonlinear multiobjective optimization, Springer, New York, USA.

[25] Deb, K., Agrawal, S., Pratap, A., Meyarivan, T. (2000). A fast elitist non-dominated sorting genetic algorithm for multi-objective optimization: NSGA-II, In: Schoenauer, M., Deb, K., Rudolph, G., Yao, X., Lutton, E., Merelo, J.J., Schwefel, H.-P. (eds), Parallel Problem Solving from Nature PPSN VI, Lecture Notes in Computer Science, Vol. 1917, Springer, Berlin, Germany, 849-858, doi: 10.1007/3-540-45356-3 83.

[26] Deb, K., Jain, H. (2014). An evolutionary many-objective optimization algorithm using reference-point-based nondominated sorting approach, part I: Solving problems with box constraints, IEEE Transactions on Evolutionary Computation, Vol. 18, No. 4, 577-601, doi: 10.1109/TEVC.2013.2281535.

[27] Hwang, C.-L., Masud, A.S.M. (1979). Multiple objective decision making - Methods and applications: A state-of-theart survey, Springer-Verlag, Berlin, Heidelberg, New York, doi: 10.1007/978-3-642-45511-7.

[28] Hassanzadeh, H.R., Rouhani, M. (2010). A multi-objective gravitational search algorithm, In: 2nd International Conference on Computational Intelligence, Liverpool, United Kingdom, 7-12, doi: 10.1109/CICSyN.2010.32.

[29] Ojstersek, R., Zhang, H., Palcic, I., Buchmeister, B. (2017). Use of heuristic Kalman algorithm for JSSP, In: Andraš. A. (ed.), XVII International scientific conference on industrial systems, Novi Sad, Faculty of Technical Sciences, Department for Industrial Engineering and Management, Novi Sad, Serbia, 72-77.

[30] Demir, Y., Kürşat Işleyen, S. (2013). Evaluation of mathematical models for flexible job-shop scheduling problems, Applied Mathematical Modelling, Vol. 37, No. 3, 977-988, doi: 10.1016/i.apm.2012.03.020.

[31] Chiang, T.-C., Lin, H.-J. (2013). A simple and effective evolutionary algorithm for multiobjective flexible job shop scheduling, International Journal of Production Economics, Vol. 141, No. 1, 87-98, doi: 10.1016/i.ijpe.2012. $\underline{03.034}$.

[32] Prester, J., Buchmeister, B., Palčič, I. (2018). Effects of advanced manufacturing technologies on manufacturing company performance, Strojniški Vestnik - Journal of Mechanical Engineering, Vol. 64, No. 12, 763-771, doi: $10.5545 /$ sv-jme.2018.5476.

[33] Lu, Y. (2017). Industry 4.0: A survey on technologies, applications and open research issues, Journal of Industrial Information Integration, Vol. 6, 1-10, doi: 10.1016/i.jii.2017.04.005. 
[34] Ojstersek, R., Buchmeister, B. (2017). Use of simulation software environments for the purpose of production optimization, In: Proceedings of the 28th DAAAM International Symposium on Intelligent Manufacturing and Automation, Zadar, Croatia, 750-758, doi: 10.2507/28th.daaam.proceedings.106.

[35] Joines, J.A., Roberts, S.D. (2015). Simulation modeling with SIMIO: A workbook, 4th Edition, CreateSpace Independent Publishing Platform, Sewickley, USA.

[36] Yang, W., Takakuwa, S. (2017). Simulation-based dynamic shop floor scheduling for a flexible manufacturing system in the industry 4.0 environment, In: Proceedings of the 2017 Winter Simulation Conference, Las Vegas, USA, 3908-3916, doi: 10.1109/WSC.2017.8248101.

[37] Dehghanimohammadabadi, M., Keyser, T.K. (2017). Intelligent simulation: Integration of SIMIO and MATLAB to deploy decision support systems to simulation environment, Simulation Modelling Practice and Theory, Vol. 71, 45-60, doi: 10.1016/j.simpat.2016.08.007. 


\section{APEM}

ISSN 1854-6250

Spletna stran: apem-journal.org Izvirni znanstveni članek

\title{
Nova metoda interaktivnega matematičnega in simulacijskega modeliranja: Študija primera na prilagodljivem terminiranju proizvodnje po naročilu
}

\author{
Ojstersek, R..$^{\mathrm{a},{ }^{*}}$, Lalic, D. ${ }^{\mathrm{b}}$, Buchmeister, B. ${ }^{\mathrm{a}}$ \\ a University of Maribor, Faculty of Mechanical Engineering, Maribor, Slovenia \\ ${ }^{b}$ University of Novi Sad, Faculty of Technical Sciences, Novi Sad, Serbia
}

\begin{abstract}
POVZETEK
V tej študiji smo preučili združljivost matematičnega in simulacijskega modela za razporejanje proizvodnih sistemov. Za ocenjevanje nove evolucijske metode računanja večkriterijskega hevrističnega Kalmanovega algoritma (MOHKA) smo uporabili matematični model problema prilagodljivega terminiranja proizvodnje po naročilu (FJSSP). Za ocenjevanje in primerjavo rezultatov optimizacije MOHKA z algoritmom večkriterijskega roja delcev (MOPSO) in večkriterijskim bare-bones algoritmom optimizacije roja delcev (BBMOPSO) je bilo uporabljenih deset standardnih testnih primerov Brandimarte in pet standardnih testnih primerov Kacem. Nabori podatkov so bili glede na njihovo zapletenost razdeljeni v tri skupine, od nizko do srednje in do visoko dimenzionalnih optimizacijskih problemov. Rezultati optimizacije MOHKA kažejo visoko primernost algoritma za reševanje kompleksnih večkriterijskih problemov, zlasti na podatkih iz realnih proizvodnih sistemov. Predstavljena je nova robustna metoda interaktivnosti optimizacijskih podatkov med matematičnim optimizacijskim algoritmom in simulacijskim modelom. Rezultati kažejo, da predstavljena metoda lahko preseže integrirano logiko odločanja komercialne simulacijske programske opreme in rezultate optimizacije prenese v simulacijski model. Našo interaktivno metodo lahko uporabimo v različnih proizvodnih in storitvenih podjetjih, da zagotovimo optimiziran in trajnosten profil stroškov in časa.
\end{abstract}

(C) 2019 CPE, University of Maribor. All rights reserved.

\section{PODATKI O ČLANKU}

Ključne besede:

Prilagodljivo terminiranje proizvodnje po naročilu;

Matematično modeliranje;

Simulacijsko modeliranje;

Interaktivnost;

Evolucijsko računanje;

Večkriterijski hevristični Kalmanov algoritem (MOHKA);

Večkriterijska optimizacija z rojem delcev (MOPSO);

Večkriterijski bare-bones algoritem optimizacije roja delcev (BBMOP-

SO)

*Kontaktna oseba: robert.ojstersek@um.si (Ojstersek, R.)

Zgodovina članka:

Prejet 15. maja 2019

Popravljen 21. novembra 2019

Sprejet 7. decembra 2019 